\title{
Phasic or terminal detrusor overactivity in women: age, urodynamic findings and sphincter behavior relationships
}

\author{
Françoise A. Valentini, Brigitte G. Marti, Gilberte Robain, Pierre P. Nelson \\ ER6 (FAV, GR, PPN), Université Pierre et Marie Curie (Paris 06), Service de MPR (FAV, GR, PPN), \\ Hôpital Rothschild and Service de Rééducation (BGM), Hôpital Saint Antoine. Paris, France
}

\begin{abstract}
Objectives: To search for relationships between phasic (P) and terminal (T) DO with age, urodynamic findings and sphincter behavior during involuntary detrusor contraction in woman.

Materials and Methods: Urodynamic studies (triple lumen catheter 7F, seated position) of 164 successive women referred for LUTS with diagnosis of DO were reviewed. Patients were stratified in 4 sub-groups: pre- (18-44 y), peri- (45$54 \mathrm{y})$, post-menopause (55-74 y) and oldest old ( $\geq 75 \mathrm{y})$. The urethral sensor was positioned at the level of the maximum

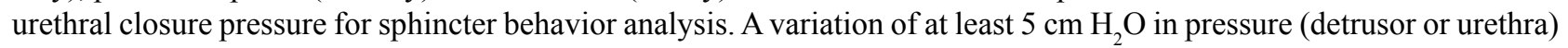
was chosen to assert DO or sphincter response. Sphincter response was classified as relaxation (re) before or during DO, or steady (st).

Results: Occurrence of P and TDO was similar: 77 P and 87 T. The PDO group was significantly younger ( $\mathrm{p}=0.0003$ ). TDO was more frequent in patients with a history of neurological disease.

The percentage of PDO remained almost constant in age groups, while that of TDO increased with age from $6.7 \%$ to $23.2 \%(\mathrm{p}=0.0013)$.

Uninhibited contraction occurred at a smaller bladder volume in the P group: $149 \pm 95 \mathrm{vs} .221 \pm 113 \mathrm{~mL}$ ( $\mathrm{p}<0.0001$ ).

Steady sphincter predominated in the TDO subgroup: $45.9 \%$ vs. $32.1 \%$ and increased significantly in each DO sub-group of $\geq 75 \mathrm{y}$.

Conclusion: Steady sphincter during both P and TDO, and occurrence of TDO appear as specific of aging. The last result could be related to structural changes in the detrusor muscle with aging.
\end{abstract}

Key words: Urinary bladder, overactive; urinary incontinence; Women; Urodynamics

Int Braz J Urol. 2011; 37: 773-780

\section{INTRODUCTION}

Detrusor overactivity (DO) is a frequent urodynamic diagnosis in women with urge syndrome with or without incontinence. DO is characterized by involuntary detrusor contraction (IDC) during the filling phase and can be idiopathic when there is no defined cause (IDO) or neurogenic when there is a relevant neurological condition (NDO) (1). According to the ICS recommendations, it is usual to distinguish phasic (P) (wave(s) with or without leakage) from terminal (T) DO (single involuntary detrusor contraction occurring at cysto- metric capacity, which cannot be suppressed, and results in incontinence, usually resulting in bladder emptying) (1). To make a distinction between $P$ and TDO is sometimes difficult: high vesical pressure or/and pain during cystometry might lead to stop the filling and to under-estimate DO. Distinction of TDO from decreased bladder compliance needs to repeat the cystometry at lower filling rate to assert or to invalidate DO.

As DO is believed to underlie overactive bladder (OAB) symptoms, most studies focus on relationships between $\mathrm{OAB}$ and $\mathrm{DO}(2-6)$. If, the impact of DO on bladder function in women has 
been widely studied (7-11), few studies have been conducted to analyze the relationships between each type of DO with age, history of neurological disease, urodynamic findings including sphincter behavior during IDC $(2,12-14)$.

Our purpose was to look for relationships between $\mathrm{P}$ or TDO and these conditions or findings.

\section{MATERIALS AND METHODS}

Between June 2005 and December 2007, 684 women were referred in our outpatient clinic for investigation of lower urinary tract (LUT) dysfunction. Nineteen were excluded (complete spinal cord injury, severe dementia (mini mental state $<20$ ) and grade $\geq 2$ prolapse). Among the 665 eligible women, the urodynamic diagnosis of DO was made in 164; of these, 72 had a history of neurological disease (Table-1). The DO population was then stratified in 4 age groups: pre- (18-44y), peri- $(45-54 y)$, postmenopause (55-74y) and oldest old ( $\geq 75 y$ ).

All patients had evaluation including medical history and usual medication, bladder diary for of the symphisis pubis. For urethral pressure recording, the catheter eye was positioned at the level of the maximum urethral closure pressure (located from a urethral profilometry bladder empty). Abdominal pressure was recorded using a punctured intra-rectal balloon catheter.

The urethral sensor was positioned at the level of the maximum urethral closure pressure (MUCP) for sphincter behavior analysis.

To avoid instrumental errors, we considered that there was an episode of DO when the detrusor pressure increased of at least $5 \mathrm{cmH}_{2} \mathrm{O}$ in pressure; in the same way, a variation of $\pm 5 \mathrm{~cm}$ $\mathrm{H}_{2} \mathrm{O}$ in urethral pressure was chosen to evaluate the sphincter response. In case of rhythmic rectal contractions, the change in vesical pressure was considered. Shifting of the urethral transducer during filling and non rhythmic rectal activity during IDC were criteria for exclusion.

One voiding cycle was recorded during each cystometry. For 18 patients a second cystometry at a lower filling rate of $20 \mathrm{~mL} / \mathrm{min}$ was performed to conclude between decreased bladder compliance and DO.

Table 1 - Description of the DO population with a history of neurological disease. In each age-group, the number of patients is presented as total (a) and in parentheses $b P$ and $c T D O: a(b P+c T)$.

\begin{tabular}{lccccc}
\hline & $\mathbf{1 8 - 4 4} \mathbf{y}$ & $\mathbf{4 5 - 5 4} \mathbf{y}$ & $\mathbf{5 5 - 7 4} \mathbf{y}$ & $\mathbf{2 7 5} \mathbf{y}$ & $\sum$ \\
\hline $\begin{array}{l}\text { Encephalic lesion (stroke, } \\
\text { meningioma) }\end{array}$ & $1(\mathrm{P})$ & $5(1 \mathrm{P}+4 \mathrm{~T})$ & $9(3 \mathrm{P}+6 \mathrm{~T})$ & $7(1 \mathrm{P}+6 \mathrm{~T})$ & $22(6 \mathrm{P}+16 \mathrm{~T})$ \\
Parkinson's disease & 0 & $1(\mathrm{~T})$ & $1(\mathrm{P})$ & $4(1 \mathrm{P}+3 \mathrm{~T})$ & $6(2 \mathrm{P}+4 \mathrm{~T})$ \\
Multiple sclerosis & $8(4 \mathrm{P}+4 \mathrm{~T})$ & $7(4 \mathrm{P}+3 \mathrm{~T})$ & $6(3 \mathrm{P}+3 \mathrm{~T})$ & $1(\mathrm{~T})$ & $22(11 \mathrm{P}+11 \mathrm{~T})$ \\
Supra-sacral spinal cord lesion & $4(2 \mathrm{P}+2 \mathrm{~T})$ & $4(1 \mathrm{P}+3 \mathrm{~T})$ & $8(3 \mathrm{P}+5 \mathrm{~T})$ & $4(1 \mathrm{P}+3 \mathrm{~T})$ & $20(7 \mathrm{P}+13 \mathrm{~T})$ \\
Sub-sacral and Peripheral & $1(\mathrm{P})$ & $1(\mathrm{P})$ & 0 & 0 & $2(2 \mathrm{P})$ \\
$\sum$ & $14(8 \mathrm{P}+6 \mathrm{~T})$ & $18(7 \mathrm{P}+11 \mathrm{~T})$ & $24(10 \mathrm{P}+14 \mathrm{~T})$ & $16(3 \mathrm{P}+13 \mathrm{~T})$ & 72 \\
\hline
\end{tabular}

at least 48 hours including voiding times and voided volumes during day- and night-time, physical examination and dipstick urinalysis.

Cystometry was performed with a triple lumen catheter $7 \mathrm{~F}$ at a filling rate of $50 \mathrm{~mL} / \mathrm{min}$ in seated position using a Dorado ${ }^{\circledR}$ unit from Laborie.

Pressures were zeroed to atmosphere with the transducers placed at the level of the upper edge
Sphincter response was classified as relaxation (re) before or during IDC, or remaining steady (st).

Recordings were reviewed independently by three investigators; good agreement occurred in up to $88 \%$ of the files. In the remaining $12 \%$, an additional interpretation was made jointly to reach a single conclusion. 
This study was conducted in accordance with the declaration of Helsinki. According to the local practice of our Ethics Committee, there is no formal Institutional Review Board approval required for retrospective studies.

\section{STATISTICAL ANALYSIS}

Data are presented as mean $\pm \mathrm{SD}$ and range. The Wilcoxon signed rank test was used for comparison of related samples, analysis of variance (ANOVA), the t-test and the Chi 2 test to compare unrelated samples. Statistical analysis was performed using SAS, version 5.0 (SAS Institute, Inc., Cary, NC). All statistical results were considered significant at $\mathrm{p}<0.05$.

\section{RESULTS}

In the whole DO population, there were 77 (46.9\%) PDO and 87 (53.1\%) TDO, with 28 and 44 women respectively who had a history of neurological disease (Table-1). A history of neurological disease was not significantly different (n.s.) between the DO sub-groups.

Main LUT symptoms were urgency with or without incontinence (118 patients) and frequency
(35 patients). Other symptoms were 5 stress urinary incontinence, 3 bedwetting and 3 incomplete retention. Incontinence was the main complaint for 123 $(75 \%)$ of the patients.

Table-2 shows the distribution in the different of both populations with DO (PDO or TDO) and without DO.

\section{Age incidence (Table-2):}

Comparing the four age groups, there was no significant incidence of age on occurrence of DO. TDO was more frequent than PDO with aging $(\mathrm{p}=$ 0.006).

In the DO population, PDO was predominant in the $18-44$ y age group $(p=0.008)$, and TDO in the $\geq 75$ y one $(p<0.0001)$ with a ratio $3 / 1$, while in the 45-54 y and 55-74 y age groups the ratio was close to 1 .

In the entire population, increase of TDO was significant with aging between age $(p=0.0036)$ while the decrease of PDO was not significant.

The PDO group was significantly younger $(52 \pm 19)$ than the TDO group $(63 \pm 16)(\mathrm{p}=0.0003)$.

There was no significant difference in age between women with or without neurological disease in DO subgroups.

Table 2: Number of women in each age group. No difference in age between the 3 groups: $P D O, T D O$ and no DO whatever the neurological history.

\begin{tabular}{lccccccc}
\hline age group & $\begin{array}{c}\text { History of neurological } \\
\text { disease }\end{array}$ & $\mathbf{1 8 - 4 4} \mathbf{y}$ & $\mathbf{4 5 - 5 4} \mathbf{y}$ & $\mathbf{5 5 - 7 4} \mathbf{~ y r}$ & $\mathbf{2 7 5} \mathbf{y}$ & $\mathbf{\Sigma}$ & mean age y \\
\hline No pts with PDO & & 28 & 13 & 24 & 12 & 77 & $52 \pm 19$ \\
& No & 20 & 6 & 14 & 9 & 49 & $51 \pm 18$ \\
\multirow{2}{*}{ No pts with TDO } & Yes & 8 & 7 & 10 & 3 & 28 & $53 \pm 21$ \\
& & 10 & 18 & 29 & 30 & 87 & $63 \pm 16$ \\
\multirow{3}{*}{ No pts without DO } & No & 4 & 7 & 14 & 13 & 38 & $60 \pm 16$ \\
& Yes & 6 & 11 & 15 & 17 & 49 & $66 \pm 17$ \\
& & 110 & 92 & 212 & 87 & 501 & $57 \pm 17$ \\
& No & 83 & 66 & 155 & 63 & 367 & $58 \pm 17$ \\
\hline
\end{tabular}

PDO was less frequent than TDO in advanced age $(p=0.006)$. PDO frequency did not change with ageing (n.s.) while TDO frequency increased ( $p=0.0013)$. 
(Table-3)

History of neurological disease (HND)

For women without HND, occurrence of each kind of DO was significantly different with age $(p=0.0114)$ with a decrease of PDO and an increase of TDO; for women with HND, no difference was observed.

In the PDO sub-group, HND was predominant in the peri- and post-menopausal age groups (53.8 and $41.6 \%$ ), while in the pre-menopause and the oldest groups the percentage of HND was close to that observed in the eligible population (28.5 and $25 \%$ vs. a mean value of $26.7 \%$ ). The frequency of HND was very high $(60.0$ and $61.1 \%)$ in the preand peri-menopausal age groups with TDO and re- in the T group: $144 \pm 95$ vs. $219 \pm 114 \mathrm{~mL}$ (p< 0.0001 ); the functional bladder capacity in group $\mathrm{P}$ $(296 \pm 105 \mathrm{~mL})$ was significantly higher than that in the $\mathrm{T}$ group $(246 \pm 121 \mathrm{~mL})(\mathrm{p}=0.0045)$. The functional capacity in the population without DO was $391 \pm 149 \mathrm{~mL}(\mathrm{p}<0.0001)$.

The detrusor pressure $\left(\mathrm{p}_{\text {det }}\right)$ was $37 \pm 25 \mathrm{~cm}$ $\mathrm{H}_{2} \mathrm{O}$ at the onset of flow (when intubated flow was obtained) in the $\mathrm{PDO}$ population and $35 \pm 19 \mathrm{cmH}_{2} \mathrm{O}$ at the onset of leakage in the $\mathbf{T}$ DO population (n.s.). In each DO sub-group, there was no significant difference between patients without $\left(39 \pm 29 \mathrm{~cm} \mathrm{H}_{2} \mathrm{O}\right)$ and those with HND $\left(31 \pm 14 \mathrm{~cm} \mathrm{H}_{2} \mathrm{O}\right)$; the same result was observed in each age group. There was no significant effect of aging on $\mathrm{p}_{\mathrm{det}}$.

Table 3 - Ratio of women with neurological disease $(N)$ in each sub group of DO and in each age group.

\begin{tabular}{lcccc}
\hline age group & $\mathbf{1 8 - 4 4} \mathbf{y}$ & $\mathbf{4 5 - 5 4} \mathbf{y}$ & $\mathbf{5 5 - 7 4} \mathbf{y}$ & $\geq \mathbf{7 5} \mathbf{y}$ \\
\hline$\%$ PNDO/ (PDO) & 28.5 & 53.8 & 41.6 & 25.0 \\
$\%$ TNDO/ (TDO) & 60.0 & 61.1 & 48.3 & 43.3 \\
$\%$ N/entire population & 24.3 & 28.4 & 26.8 & 27.3 \\
\hline
\end{tabular}

mained at a significant level in the other age groups (48.3 and $43.3 \%)$.

In the NDO population, neurological disorder secondary to supra-pontine (SP) lesions (encephalic lesion and Parkinson's disease) was observed in $8 / 28(28 \%)$ of the PDO group and 20/44 (45\%) of the TDO group. The distribution of SP lesions showed an increase with ageing in the TDO group.

\section{Urodynamic findings}

The bladder volume of occurrence of the first IDC in P group was smaller than that at the IDC
The maximum urethral closure pressure (MUCP) decreased with aging but was most often found normal or higher (Table-4) compared with the value expected for age $[(110-$ age $) \pm 20 \%$ in $\mathrm{cm}$ $\left.\mathrm{H}_{2} \mathrm{O}\right](15)$. Mean expected value was $62.8 \pm 22.3 \mathrm{~cm}$ $\mathrm{H}_{2} \mathrm{O}$ when the mean observed value was $69.9 \pm 37.4$ $\mathrm{cm} \mathrm{H}_{2} \mathrm{O}(\mathrm{p}=0.0021)$.

\section{tion (Table-5)}

IDC characteristics in the PDO popula-

The number of IDC varied from 1 to $>5$ with 12 leakage-micturitions during the last IDC in

Table 4: Comparison of the maximal urethral closure pressure (MUCP) with the expected value for age in each DO group (women with or without a history of neurological disease (HND)).

\begin{tabular}{lcccccc}
\hline \multicolumn{7}{c}{ P } \\
MUCP & with HND & without HND & $\Sigma$ & with HND & without HND & $\Sigma$ \\
Low & 4 & 6 & $10(12.9 \%)$ & 1 & 7 & $8(9.2 \%)$ \\
Normal & 7 & 17 & $24(31.2 \%)$ & 20 & 19 & $39(44.8 \%)$ \\
High & 17 & 26 & $43(55.8 \%)$ & 23 & 16 & $39(44.8 \%)$ \\
\hline
\end{tabular}


Table 5 - Spatio-temporal characteristics of the successive IDC in the PDO population; comparison between women with or without history of neurological disease (HND).

\begin{tabular}{lccc}
\hline & Duration (s) & Time to maximum (s) & Amplitude (cm Ho) \\
\hline Pts without HND & $14.9 \pm 1.7$ & $6.6 \pm 0.5$ & $11.1 \pm 3.3$ \\
Pts with HND & $16.1 \pm 1.8$ & $7.5 \pm 1.1$ & $14.3 \pm 1.9$ \\
$\mathrm{p}$ & n.s. & n.s. & n.s. \\
\hline
\end{tabular}

the NDO sub-group and 16 in the IDO sub-group.

Characteristics of the IDC are given in the

Table-3. There was no significant variation in the duration, time to maximum and amplitude whatever the neurological status.

Recorded sensation (desire to void) showed mainly an occurrence of the first IDC before or at the first desire to void (FDV) $(\mathrm{p}=0.033)$ (Table-6). That behavior was independent of a HND.

\section{Sphincter behavior (Table-7)}

Steady sphincter was predominant in the TDO subgroup: $45.9 \%$ vs. $32.1 \%$ (n.s.). In each DO sub-group, the ratio of steady sphincter increased in the $\geq 75$ y sub-group (P: $50.0 \%$ vs. $32-33-36 \%$; T: $65 \%$ vs. $40-35-34 \%$ ); in the same way, the ratio of sphincter relaxation before IDC decreased in the $\geq$ 75 y sub-group (P: 0\% vs. $29-42-23 \%$; T: $17 \%$ vs. 33-29-34\%).

\section{DISCUSSION}

The distinction between IDO and NDO has been frequently studied $(16,17)$, but scarce data exist regarding the conditions underlying occurrence and expression of $\mathrm{P}$ or TDO. Occurrence of TDO has been related to aging and particularly to elderly patients with neurological lesions such as cerebro- vascular accident $(3,12,18)$. In his study, Tong $(14)$ reported a high incidence of TDO with abnormal bladder sensation and unfelt PDO in men with benign prostatic hyperplasia.

Our purpose was to search for a condition which could be an indicator for occurrence of $\mathrm{P}$ or TDO. The first idea was to test aging because it is well known that LUT function changes in elderly patients. Because about half of the population (44\%) had DO and a history of neurological disease, that last condition was also analyzed. Finally, because DO is a urodynamic diagnosis, some urodynamic parameters $\left(\mathrm{p}_{\mathrm{det}}\right.$ at the onset of flow for PDO, or at the onset of leakage for TDO, MUCP and sphincter behaviour during IDC) have been investigated. In their paper, Romanzi et al. (19) compared patients (men and women) according to the main presenting symptom and the neurological status and concluded that the characteristics of the cystometric tracing during IDC were not distinct to aid in differential diagnosis but might have prognostic and therapeutic significance.

Occurrence of TDO is significantly associated with aging; an inversion of the ratio $\mathrm{PDO} / \mathrm{TDO}$ from $3: 1$ to is also observed. That result is consistent with an association between TDO and changes in LUT function due to aging. TDO would more probably due to alteration in detrusor function or abnormal micturition reflex than to sphincter incompe-

Table 6 - Recorded sensation on UDS at occurrence of the first IDC in the PDO group (FDV: first desire to void; NDV: normal desire to void; SDV: strong desire to void). The first IDC occurs significantly before or at the first desire to void $(F D V)(p=0.033)$.

\begin{tabular}{lccccc}
\hline Age group 1rst IDC & $\mathbf{1 8 - 4 4} \mathbf{y}$ & $\mathbf{4 5 - 5 4} \mathbf{y}$ & $\mathbf{5 5 - 7 4} \mathbf{y}$ & $\mathbf{2 7 5} \mathbf{y}$ & $\mathbf{\Sigma}$ \\
\hline Up to FDV & $14(50 \%)$ & $4(31 \%)$ & $11(46 \%)$ & $7(58 \%)$ & 36 \\
> FDV to NDV & 7 & 2 & 5 & 1 & 15 \\
> NDV to SDV & 7 & 7 & 8 & 4 & 26 \\
\hline
\end{tabular}


Table 7 - Relationship between sphincter behavior and a history of neurological disease (HND) at IDC in each DO group. Sphincter relaxation before or during IDC is significantly observed in PDO women without HND $(p=0.0045)$.

\begin{tabular}{lcccccc}
\hline & \multicolumn{3}{c}{ PDO } & & TDO \\
\hline Sphincter behavior & With HND & Without HND & $\Sigma$ & With HND & Without HND & $\Sigma$ \\
Relaxation before IDC & 4 & 14 & $18(24.33 \%)$ & 12 & 11 & $23(27.05 \%)$ \\
Relaxation during IDC & 8 & 21 & $29(34.52 \%)$ & 13 & 10 & $23(27.05 \%)$ \\
Steady during IDC & 15 & 12 & $27(32.14 \%)$ & 19 & 20 & $39(45.88 \%)$ \\
\hline
\end{tabular}

tence because, in the oldest group, $\mathrm{p}_{\operatorname{det}}$ at leakage is significantly lower than MUCP ( $\mathrm{p}<0.0001)$. With regard to aging-related $\mathrm{DO}$, a characteristic structural pattern (20) and age-related changes in muscarinic receptor functions have been reported (21) but not related to each kind of DO.

The percentage of women with PDO is significantly higher than that of TDO in the 18-44 y subgroup $(\mathrm{p}=0.008)$. A hypothesis to explain that result could be a high incidence of spinal lesions (spina bifida, multiple sclerosis) in younger. Unfortunately, in this age group the number of spinal lesion is the same in P and TDO groups.

Among the whole population, occurrence of DO was higher in the group with history of neurological disease ( $44 \%$ vs. $18 \%$ ) as it has been previously reported (17-22). It has been verified that there was no bias of recruitment, as the percentage of patients with a neurological disease was similar in the different age groups: $26.8 \pm 1.3 \%$. Aging and history of neurological disease appear to be conditions for occurrence of TDO; consequently, the role of encephalic lesions (stroke, meningioma and Parkinson's disease) which frequency increases with ageing is a question. Unfortunately, that sub-group comprises only nine women, which is too small a group to conclude.

As expected, the functional bladder capacity (FBC) is lower in the DO population for which main motive for urodynamics is urge or mixed incontinence compared to the population without DO.

Comparing $\mathrm{P}$ and TDO patients, $\mathrm{FBC}$ is significantly higher in the PDO group. That result is consistent with the ability of PDO patients to counterbalance the increase in detrusor pressure and to prevent voiding. $p_{\text {det }}$ does not differ at the onset of flow for PDO women and at the onset of leakage for
TDO women. That result confirms the inability to abort IDC for TDO women. For our female population, the values of $\mathrm{p}_{\text {det }}$ at IDC are lower than the values reported by Romanzi et al. (19) whose study included men with urethral obstruction leading to detrusor hypertrophy.

MUCP decreases with aging but its value remains normal and higher the value expected for the age which can be the consequence of long-lasting DO and unconscious reinforcement of the striated sphincter.

An intuitive proposal that the larger the amplitude of the IDC, the more severe DO has been invalidated by Miller et al. (23), as it is severely confounded by the urethral sphincter function. That result is consistent with our findings; we do not observe differences between duration, time to maximum, and amplitude in successive IDC whatever the neurological status, and in addition, MUCP is normal or higher than the expected value for age. An unexplained result is the value of detrusor pressure generated during IDC which is much lower than the values reported by Romanzi et al. (19). In his paper (2), P Abrams suggests that PDO tends to be characterized by contractions of increasing amplitude as the bladder volume increases and that this pattern is seen in most IDO of middle age men and women. We don't observe any increase of the IDC amplitude with bladder filling. The number of IDC in the PDO group is lower for the NDO group (only $28 \%$ had 3 IDC vs. $43 \%$ in the IDO group); that result could be a limitation of the comparison between characteristics (duration, time to maximum, and amplitude) of the IDC between NDO and IDO but in this study there is no significant difference between these parameters during the first and second IDC. 
In the PDO group, the first IDC is mainly observed up to first desire to void, which could imply abnormal intrinsic bladder reflexes (24) to bladder filling.

Incidence of steady sphincter in the oldest old group might be the consequence of aging on urethral function, as the threshold of urethral sensation increased in the elderly (3). The same conclusion can be proposed for the decrease of sphincter relaxation before IDC with aging.

In the population with a history of neurological disease, one observes a higher occurrence of a steady sphincter during IDC in the PDO group and a lower occurrence of sphincter relaxation before IDC when the neurological condition is an incomplete spinal cord injury.

Our study has several limitations, including its retrospective nature as well as the limited number of patients in each type of neurological lesion; its merit is that it is to be the first report assessing to find characteristics for each kind of DO.

\section{CONCLUSIONS}

Steady sphincter during non-inhibited detrusor contraction for both $\mathrm{P}$ and TDO, and occurrence of TDO appear as specific of aging. In the elderly, occurrence of a steady sphincter may be associated with loss of sensory nerve function in the urethra and occurrence of TDO could be related to the structural changes in the detrusor muscle with aging. Looking at a history of neurological disease, there is a trend of increase for both P and TDO with aging, except in the oldest group where TDO predominates.

\footnotetext{
ABBREVIATIONS:

DO $=$ Detrusor Overactivity

FBC $=$ Functional Bladder Capacity

FDV $=$ First Desire to Void

HND $=$ History of Neurological Disease

IDC $=$ Involuntary Detrusor Contraction

IDO $=$ Idiopathic Detrusor Overactivity

LUT $=$ Lower Urinary Tract

MUCP = Maximum Urethral Closure pressure

NDO $=$ Neurogenic Detrusor Overactivity

$\mathbf{N D V}=$ Normal Desire to Void
}

$\mathbf{O A B}=$ Overactive Bladder

$\mathbf{p}_{\text {det }}=$ Detrusor pressure

PDO $=$ Phasic Detrusor Overactivity

STV $=$ Strong Desire to Void

TDO $=$ Terminal Detrusor Overactivity

\section{CONFLICT OF INTEREST}

None declared.

\section{REFERENCES}

1. Abrams P, Cardozo L, Fall M, Griffiths D, Rosier P, Ulmsten $U$, et al.: The standardisation of terminology of lower urinary tract function: report from the Standardisation Sub-committee of the International Continence Society. Neurourol Urodyn. 2002; 21: 167-78.

2. Abrams P: Describing bladder storage function: overactive bladder syndrome and detrusor overactivity. Urology. 2003; 62 (5 Suppl 2): 28-37; discussion 40-2.

3. Guralnick ML, Grimsby G, Liss M, Szabo A, O'Connor RC: Objective differences between overactive bladder patients with and without urodynamically proven detrusor overactivity. Int Urogynecol J Pelvic Floor Dysfunct. 2010; 21: 325-9.

4. Hashim H, Abrams P: Overactive bladder: an update. Curr Opin Urol. 2007; 17: 231-6.

5. Schaefer W: Re: Is the bladder a reliable witness for predicting detrusor overactivity? $\mathrm{H}$. Hashim and P. Abrams. J Urol, 175: 191-195, 2006. J Urol. 2006; 176: 1255-7; author reply 1256-7.

6. Andersson KE: Mechanisms of Disease: central nervous system involvement in overactive bladder syndrome. Nat Clin Pract Urol. 2004; 1: 103-8.

7. Pfisterer MH, Griffiths DJ, Schaefer W, Resnick NM: The effect of age on lower urinary tract function: a study in women. J Am Geriatr Soc. 2006; 54: 405-12.

8. Kayigil O, Metin A, Atmaca AF: Obstructive urodynamic findings in idiopathic detrusor overactivity. Int Urol Nephrol. 2007; 39: 445-8.

9. Garnett S, Swithinbank L, Ellis-Jones J, Abrams P: The long-term natural history of overactive bladder symptoms due to idiopathic detrusor overactivity in women. BJU Int. 2009; 104: 948-53.

10. Serati M, Salvatore S, Cattoni E, Soligo M, Cromi A, Ghezzi F: Ultrasound measurement of bladder wall thickness in different forms of detrusor overactivity. Int Urogynecol J Pelvic Floor Dysfunct. 2010; 21: 1405-11. 
11. Lee SR, Kim HJ, Kim A, Kim JH: Overactive bladder is not only overactive but also hypersensitive. Urology. 2010; 75: 1053-9.

12. Kessler TM, Madersbacher H: Urodynamic phenomena in the aging bladder. Urologe A. 2004; 43: 542-6.

13. Schaefer W, Tadic S, Griffiths DJ, Resnick NM: Overactive bladder and detrusor overactivity: but what does the sphincter do? J Urol 2007;177(suppl 4):556. \#abstract 1677.

14. Tong YC: Comparisons of urodynamic findings and voiding habits in patients with concomitant benign prostatic hyperplasia and detrusor overactivity presenting with or without the symptom of urgency. Urol Int. 2007; 78: 219-25.

15. Constantinou CE: Urethrometry: considerations of static, dynamic, and stability characteristics of the female urethra. Neurourol Urodyn. 1988; 7: 521-39.

16. Defreitas GA, Lemack GE, Zimmern PE, Dewey RB, Roehrborn CG, O'Suilleabhain PE: Distinguishing neurogenic from non-neurogenic detrusor overactivity: a urodynamic assessment of lower urinary tract symptoms in patients with and without Parkinson's disease. Urology. 2003; 62: 651-5.

17. Lemack GE, Frohman EM, Zimmern PE, Hawker $\mathrm{K}$, Ramnarayan P: Urodynamic distinctions between idiopathic detrusor overactivity and detrusor overactivity secondary to multiple sclerosis. Urology. 2006; 67: 960-4.

18. Geirsson G, Fall M, Lindström S: Subtypes of overactive bladder in old age. Age Ageing. 1993; 22: 125-31.

19. Romanzi LJ, Groutz A, Heritz DM, Blaivas JG: Involuntary detrusor contractions: correlation of urodynamic data to clinical categories. Neurourol Urodyn. 2001; 20: 249-57.
20. Elbadawi A, Yalla SV, Resnick NM: Structural basis of geriatric voiding dysfunction. II. Aging detrusor: normal versus impaired contractility. J Urol. 1993; 150: 1657-67.

21. Andersson KE: Antimuscarinic mechanisms and the overactive detrusor: an update. Eur Urol. 2011; 59: 377-86.

22. Ashok K, Wang A: Detrusor overactivity: an overview. Arch Gynecol Obstet. 2010; 282: 33-41.

23. Miller KL, DuBeau CE, Bergmann M, Griffiths DJ, Resnick NM: Quest for a detrusor overactivity index. J Urol. 2002; 167: 578-84; discussion 584-5.

24. Fowler CJ: Integrated control of lower urinary tract-clinical perspective. Br J Pharmacol. 2006; 147(Suppl 2): S14-24.

Submitted for publication:

January 04, 2011

Accepted after revision:

April 05, 2011 